\title{
Vestibulo-ocular reflex abnormality in Vogt-Koyanagi-Harada syndrome
}

\author{
Hidehiro Oku, Satoshi Ishikawa
}

\begin{abstract}
The eye movements of 15 patients with VogtKoyanagi-Harada (VKH) syndrome were analysed. Transient dizziness occurred in $\mathbf{6 0 \%}$ of the patients. Eight patients (53\%) exhibited horizontal jerk nystagmus that was completely inhibited by visual fixation. Eight cases (53\%) exhibited the elevation of vestibulo-ocular reflex (VOR) gain in darkness. Six of the patients with nystagmus had defective smooth pursuit movements with reduced gain on the side ipsilateral to the nystagmus. Saccadic and optokinetic eye movements were intact, and visual vestibular interaction was also normal. Impaired eye movements in VKH syndrome may be caused by asymmetry of the vestibular function secondary to the labyrinthine inflammation.
\end{abstract}

(Br F Ophthalmol 1994; 78: 912-916)

Vogt-Koyanagi-Harada (VKH) syndrome is a bilateral progressive panuveitis with exudative retinal detachment that is associated with vitiligo, alopecia, and poliosis. ${ }^{1-4}$ An immune response involving melanocytes is considered to be a major cause of this syndrome. ${ }^{4}$ Because of the embryogenetic, morphological, and functional similarity between the uveal membrane and the leptomeninges, ${ }^{5}$ the central nervous system (CNS) may be involved in VKH syndrome, generating pleocytosis with an increase of protein in the cerebrospinal fluid (CSF). ${ }^{45}$ Neurological symptoms such as headache, nausea, dysacousia, and dizziness are common complaints of patients with VKH syndrome, and the findings may include optic neuritis, ${ }^{4}$ hemiplegia, ${ }^{5}$ and tonic pupil. ${ }^{6}$ Therefore, this syndrome has been referred to as a uveomeningitis or uveoencephalitis. ${ }^{5}$ Despite extensive clinical studies, there has been no detailed quantitative investigation of ocular movements in $\mathrm{VKH}$ syndrome and the precise lesion responsible for dizziness is not known. It seems possible that analysis of eye movements, including VOR may detect CNS lesions in patients with VKH syndrome. Thus, in the present study, we analysed the eye movements of 15 patients with $\mathrm{VKH}$ syndrome.

\section{Subjects and methods}

PATIENTS

We studied 15 patients with VKH syndrome treated at the Department of Neuro-Ophthalmology of Kitasato University Hospital. The patients included six males and nine females aged from 17 to 58 years, with a mean of 35.9 years. Inclusion criteria were (1) bilateral uveitis, (2) focal subretinal leakage from the choroid, and (3) evidence of CSF inflammation.

\section{AUDIOMETRY}

Auditory function was examined using pure tone audiometry. Measurements were performed at $500 \mathrm{~Hz}$ and $1,2,4$, and $8 \mathrm{kHz}$, because the most important range of hearing for speech is between $300 \mathrm{~Hz}$ and $3 \mathrm{kHz}$, while testing higher frequencies can be useful to detect early impairment.

\section{RECORDING OF EYE MOVEMENTS}

Horizontal and vertical eye movements were recorded using direct current electro-oculography (DC-EOG). The recordings were simultaneously made with a thermal array polygraph and a rectilinear pen system, stored on FM tape, and analysed on line by a digital computer. There was no previous history of strabismus, ophthalmoplegia, or otological disorders in any of the patients before the diagnosis of $\mathrm{VKH}$ syndrome. In addition, all patients were examined specifically by an otologist to exclude previous middle or inner ear damage. Despite their impaired vision, all patients were able to maintain fixation on a $5 \mathrm{~mm}$ diameter red diode target at a distance of $200 \mathrm{~cm}$. If spontaneous nystagmus was noted, arithmetical calculation was added. Systemic administration of corticosteroids was not begun before the oculomotor examination.

\section{VESTIBULO-OCULAR REFLEX}

The vestibulo-ocular reflex (VOR) was stimulated by sinusoidal whole body rotation around the vertical axis using an electrically driven rotating chair (Nagashima Co SRA 2, Tokyo). Patients were seated in the chair with the head inclined 30 degrees below the horizontal and held by a chin rest as well as occipital supports with a head band. The head position was monitored by a potentiometer coupled to the base of the chair. The chair rotated sinusoidally through an arc of 60 degrees at peak velocities of 30, 60, and 90 degrees per second, equally frequencies of $0.16,0.32$, and $0.47 \mathrm{~Hz}$, respectively. The VOR gain values were computed as the ratio of the peak smooth eye movement velocity to the peak head velocity. Measurements were taken under three different sets of conditions in the following order: (1) in darkness, (2) during fixation on a stationary red diode target with a diameter of $5 \mathrm{~mm}$ positioned $200 \mathrm{~cm}$ from the patient (dot on wall), and (3) during fixation on 


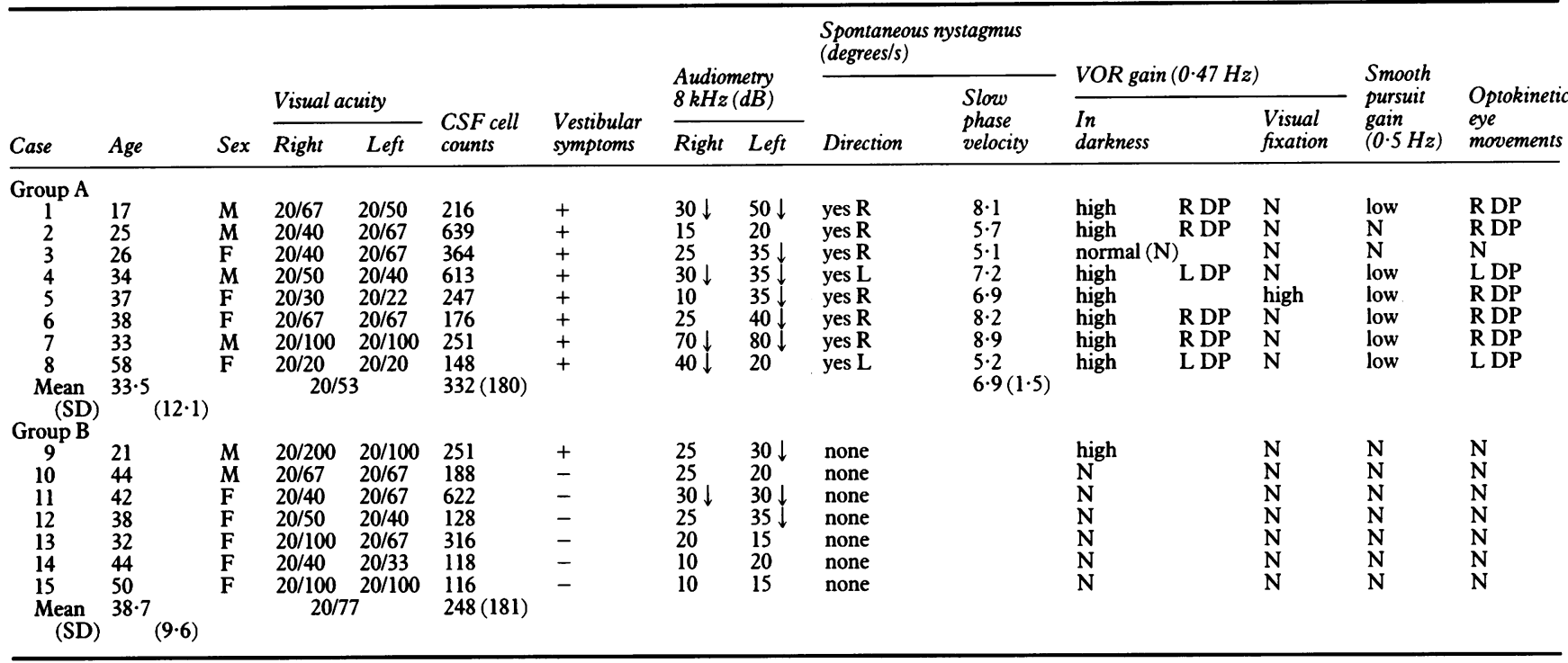

Right or left indicates the fast phases of spontaneous nystagmus, the VOR, optokinetic nystagmus, and eye tracking in smooth pursuit.

the same red diode attached to the chair $50 \mathrm{~cm}$ in front of the patient and moving with the head (visual fixation).

For each patient, the VOR gain was measured at each frequency under the three sets of conditions given above. Individual mean gain values

\section{(A) Spontaneous nystagmus}

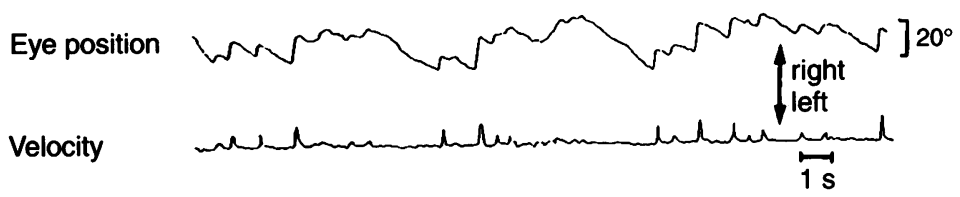

(B) VOR in darkness

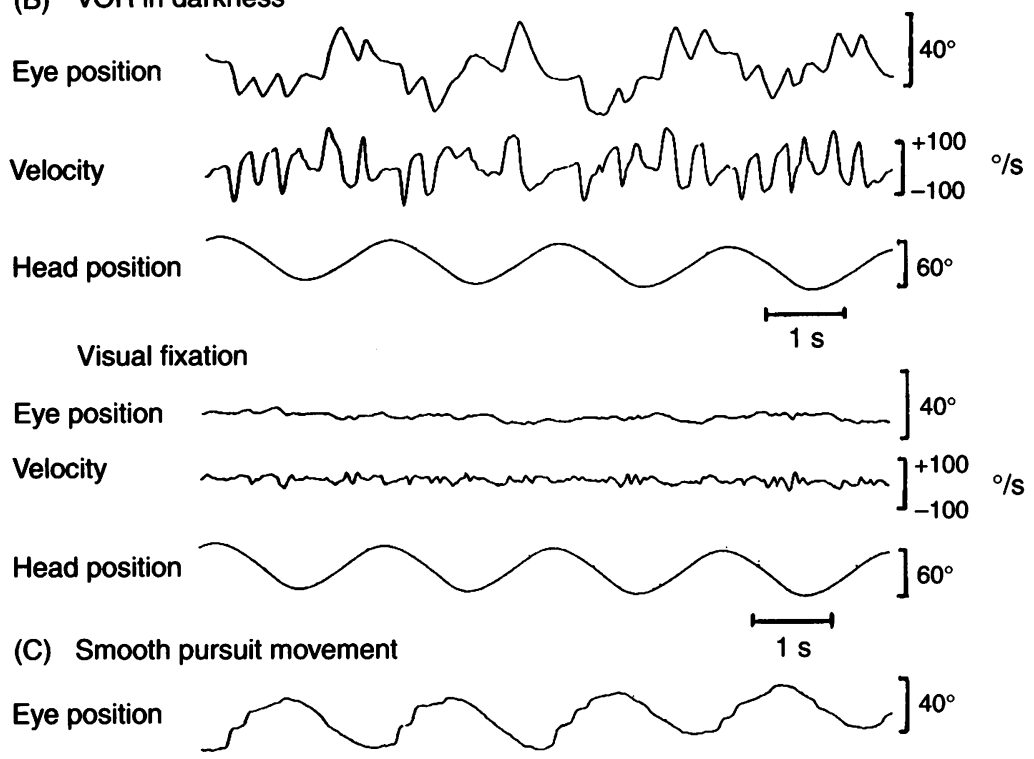

Velocity

Target

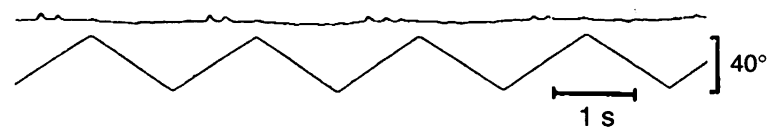

Figure 1 Typical recording of eye movements (case 6). The right eye recording is shown. In each recording, an upward deflection indicates movement to the right and vice versa.

(A) Spontaneous nystagmus to the right with Frenzel's glasses. (B) Horizontal vestibulo-ocular reflex $(V O R)$ induced by sinusoidal rotation of $0.47 \mathrm{~Hz}$. Hyperactivity was observed in darkness. The induced nystagmus was almost completely suppressed by visual fixation.

(C) Horizontal smooth pursuit movements. The target velocity was 40 degrees/second. Gain to the right was reduced to 0.86 . were determined from the optimum data obtained from a series of more than 30 sinusoids.

A modified version of the visual suppression test $^{8}$ for caloric nystagmus was used to quantify the visual suppression ratio of the VOR (calculated as the slow phase velocity (SPV) of the 'VOR in darkness' minus the SPV of 'visual fixation', divided by the SPV of the 'VOR in darkness'). This ratio was then multiplied by 100 to give the percentage visual suppression. The frequency of rotation was $0.47 \mathrm{~Hz}$.

\section{CALORIC TEST}

In order to examine vestibular function on each side independently, we performed the caloric test using $5 \mathrm{ml}$ of cold water at $5^{\circ} \mathrm{C}$, and measured the duration and the maximum slow phase velocity of the induced nystagmus.

\section{SMOOTH PURSUIT EYE MOVEMENTS}

Horizontal smooth ocular pursuit movements with an amplitude of $\mathbf{4 0}$ degrees were measured. The target moved at a constant velocity of $24 \cdot 2$ and then 40 degrees per second, equivalent to 0.3 and $0.5 \mathrm{~Hz}$, respectively. The patient was required to follow the target as accurately as possible. Ten trajectories were selected and averaged for each patient when the gain was calculated.

\section{SACCADIC EYE MOVEMENT}

The 15 patients underwent a quantitative study of saccadic ocular movements with an amplitude of 20 degrees. The velocity and latency of each saccade were determined. Presentation of the fixation target was done at random intervals and 10 saccadic movements were averaged for each patient to determine the mean velocity.

\section{OPTOKINETIC EYE MOVEMENT}

Patients were seated at the centre of a horizontally rotating drum with a diameter of $150 \mathrm{~cm}$ and 
VOR in darkness $\quad 7$ July

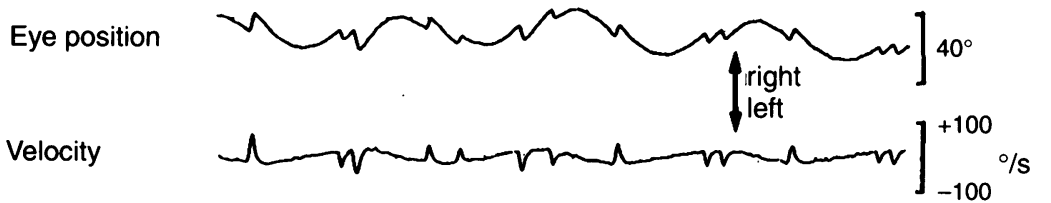

Head position

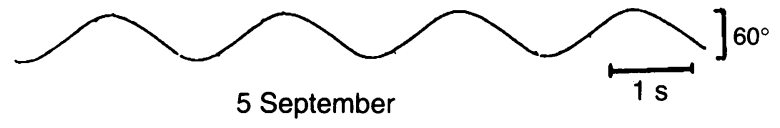

Eye position

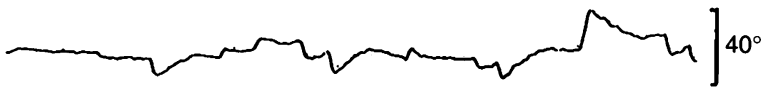

Velocity

Head position

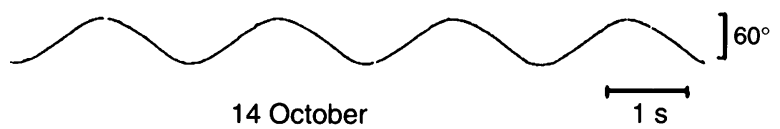

Eye position

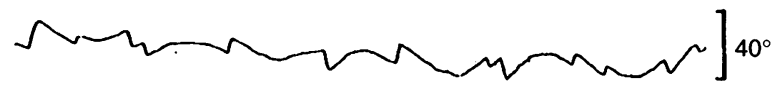

Velocity

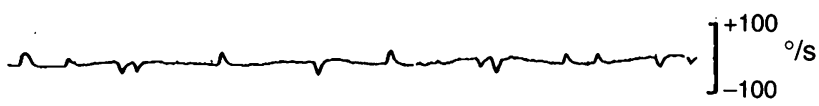

Head position

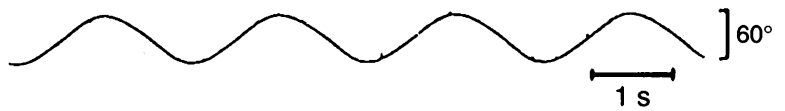

Figure 2 Transient deterioration of the vestibulo ocular reflex (VOR) in case 3. Rotation was sinusoidal at $0.47 \mathrm{~Hz}$ in each recording. The induced nystagmus was small on 5 September, but returned to its previous level by 14 October. a vertical height of $200 \mathrm{~cm}$. The drum was painted with $3 \mathrm{~cm}$ vertical black stripes spaced at $27 \cdot 7$ degree intervals on a white background, and it was rotated in the clockwise and anticlockwise directions to produce optokinetic stimulation of the entire visual field. The velocity of rotation was increased with uniform acceleration from 0 to 180 degrees per second over 45 seconds, and then returned to 0 degrees 45 seconds later. Induced nystagmus and its differentials were recorded in each direction (optokinetic pattern test $^{9}$ ). In addition, the number of cycles of nystagmus and the slow phase velocity were measured and compared in each direction.

Normal data on the various eye movements were obtained from 20 age matched healthy subjects (mean age 32.5 years). Gain values more than 2 SD away from the normal mean values were considered to be abnormal.

\section{Results}

\section{CLINICAL FEATURES}

The clinical features and the eye movement data are summarised in Table 1 . The visual acuity was reduced to less than 20/20 in all patients except case 8. All of the patients showed lymphocytic (range $87-100 \%$ ) pleocytosis $>100 \times 10^{6} / 1$ in the CSF. The CSF protein concentration was also slightly increased and the average level was 62.5 $\mathrm{mg} / \mathrm{dl}$ (range 40-87 mg/dl).

Nine patients (cases 1 to 9 ) (60\%) complained of dizziness and vertigo, which improved on closing the eyes, an indication of its vestibular origin. These vestibular symptoms occurred 5-7 days after the onset of visual impairment.

Hearing was impaired at high frequencies and was below $30 \mathrm{~dB}$ at $8 \mathrm{kHz}$ in 10 patients $(67 \%)$, indicated by arrows in Table 1 . Auditory recruit- ment was recognised in two patients (cases 1 and 5).

SPONTANEOUS NYSTAGMUS

The patients could be divided into two groups, (a) with spontaneous nystagmus, and (b) without nystagmus. A representative recording of eye movements from case 6 is shown in Figure 1. Spontaneous nystagmus was of the horizontal jerk type (Fig 1A). The quick phase was to the right in six patients, and to the left in two. Nystagmus was more obvious in the dark and under Frenzel's glasses. It was enhanced by arithmetic calculation and was inhibited by fixation. The mean amplitude was $7 \cdot 3$ degrees and the frequency was $0.99 \mathrm{~Hz}$.

\section{VESTIBULO-OCULAR REFLEX}

The VOR in darkness was hyperactive (Fig 1B). Gain elevation was seen in eight patients (53\%) (Table 1). Any right or left sided disparity in gain of more than $0 \cdot 10$ was considered to indicate a directional preponderance and was noted in six patients. The direction of the nystagmus and side dominance of VOR gain were well correlated (cases 1, 2, 4, 6, 7, and 8) (Table 1).

While fixating on a stationary target (dot on wall), the normal subjects and patients showed a VOR gain near unity throughout the velocity range. During attempted fixation on a moving target (visual fixation), VOR gain was effectively suppressed in all cases (Fig 1B). The visual suppression rate for the patients was $78.7 \%$, while that for the normal subjects was $77 \cdot 5 \%$. (Case 5 showed elevated VOR gain with visual fixation. However, her visual suppression rate was $78 \%$ and considered to be normal.)

Case 1 showed marked VOR directional preponderance to the right in darkness. Caloric stimulation revealed left canal paresis. Case 3 had normal vestibular function during VOR assessment (Fig 2, 7 July) and caloric stimulation at the initial examination. However, after a severe recurrence, the VOR in darkness was transiently reduced (Fig 2, 5 September). Canal paresis was not seen in the other patients.

\section{SMOOTH PURSUIT EYE MOVEMENTS}

Six patients with nystagmus showed defective smooth pursuit movements with reduced gain at the target velocity of $0.5 \mathrm{~Hz}$ (Table 1 ). Gain was reduced upon tracking in the same direction as the nystagmus (Fig 1C).

\section{SACCADIC EYE MOVEMENT}

The mean velocities were both normal for horizontal saccadic eye movements to 20 degrees in the temporal and nasal directions from the midline. None of the patients exhibited abnormal saccadic trajectories. The saccadic latency time was within $250 \mathrm{~ms}$ in all patients and this was considered to be within normal limits.

\section{OPTOKINETIC EYE MOVEMENTS}

Optokinetic nystagmus was well induced in all 
Table 2 Gain of vestibulo-ocular reflex and smooth pursuit movements

\begin{tabular}{|c|c|c|c|c|}
\hline & \multicolumn{2}{|c|}{ Group A patients $(n=8)$} & \multirow{2}{*}{$\underset{(n=7)}{\text { Group B patients }}$} & \multirow{2}{*}{$\begin{array}{l}\text { Normal subject } \\
(n=20)\end{array}$} \\
\hline & Ipsilateral & Contralateral & & \\
\hline \multicolumn{5}{|c|}{ Gain of vestibulo-ocular reflex (mean (SD)) } \\
\hline \multicolumn{5}{|c|}{ In darkness } \\
\hline $0.16 \mathrm{~Hz}$ & $0.88(0.25)^{\star}$ & $0.78(0 \cdot 18)^{\star}$ & $0 \cdot 70(0 \cdot 18)$ & $0.64(0 \cdot 12)$ \\
\hline $0.32 \mathrm{~Hz}$ & $0.90(0.22)^{\star}$ & $0.88(0.18)^{\star}$ & $0.75(0 \cdot 16)$ & $0.65(0 \cdot 10)$ \\
\hline $0.47 \mathrm{~Hz}$ & $1 \cdot 14(0 \cdot 20)^{\star}$ & $0.94(0.19)^{\star}$ & $0.73(0.09)$ & $0.73(0.09)$ \\
\hline \multicolumn{5}{|l|}{ Visual fixation } \\
\hline $0.47 \mathrm{~Hz}$ & $0.21(0.05)$ & $0 \cdot 19(0 \cdot 06)$ & $0 \cdot 17(0 \cdot 04)$ & $0 \cdot 17(0.05)$ \\
\hline Suppression (\%) & $81 \cdot 2(4 \cdot 9)$ & $80 \cdot 2(4 \cdot 7)$ & $76 \cdot 4(4 \cdot 6)$ & $77 \cdot 5(5 \cdot 4)$ \\
\hline \multicolumn{5}{|c|}{ Gain of smooth pursuit movements (mean (SD)) } \\
\hline $0.3 \mathrm{~Hz}$ & $0.88(0.07)^{\star}$ & $1.02(0.05)$ & $0.98(0.04)$ & $1.01(0.03)$ \\
\hline $0.5 \mathrm{~Hz}$ & $0.86(0 \cdot 10)^{\star}$ & $0.94(0.04)$ & $0.95(0.05)$ & $0.99(0.04)$ \\
\hline
\end{tabular}

${ }^{\star} \mathrm{p}<0.05$ (Mann-Whitney test).

Ipsilateral or contralateral means the direction of the fast phase of the VOR, the direction of eye tracking was the same or opposite to that of spontaneous nystagmus.

patients and there was no reduction in the number of cycles of induced nystagmus when compared with the normal subjects. Seven patients exhibited directional preponderance, with a disparity in the maximum slow phase velocity of more than 10 degrees/second, and it was in the same direction as detected by the VOR in darkness.

\section{CLINICAL COURSE}

Vestibular symptoms and meningism was resolved rapidly with steroid treatment $(120-150$ $\mathrm{mg}$ of prednisolone as an initial dose) within a week. However, spontaneous nystagmus in the dark persisted for 2-3 weeks. The steroid dose gradually decreased over a 2 month period, and after treatment all of the patients had a visual acuity over 20/20 except case 3. Asymmetric vestibular function disappeared, while the VOR and smooth pursuit gain also returned to normal.

\section{CLINICAL CORRELATIONS}

The patients with spontaneous nystagmus (group A) and those without nystagmus (group B) had quite different clinical features. Vestibular symptoms and high frequency deafness at $8 \mathrm{kHz}$ were prominent in group $\mathrm{A}$. The VOR and smooth pursuit gain of the patients is compared with that of the normal controls in Table 2 using the Mann-Whitney test. Ipsilateral VOR gain in darkness was higher than contralateral gain, but both parameters were elevated $(p<0.05)$. In addition, ipsilateral smooth pursuit gain was reduced $(\mathrm{p}<0.05)$. In contrast, visual suppression of the VOR was not impaired. There were no significant differences between group $B$ patients and the normal subjects. However, VOR gain in darkness for the 15 patients as a whole was still elevated $(p<0 \cdot 05,0 \cdot 32-0 \cdot 47 \mathrm{~Hz})$.

\section{Discussion}

This study showed that some patients with $\mathrm{VKH}$ syndrome suffer from vestibular symptoms and exhibit oculomotor abnormalities. Abnormalities were seen in slow eye movements, while saccadic and quick movements were less affected. The spontaneous nystagmus had characteristics typical of peripheral vestibular nystagmus. ${ }^{10}$ Directional preponderance of the VOR in darkness reflected an imbalance of tonic neural activity between the vestibular nuclei ${ }^{10}$ and the slow phase drift was towards the side with lower VOR gain. High frequency deafness was also found in these patients and auditory recruitment was noted. These results suggest that both the cochlear and vestibular nerves were simultaneously involved, with the origin of the hearing impairment being inner ear pathology. A $\mathrm{T}$ cell mediated immune reaction to melanocytes is a major cause of VKH syndrome." Melanocytes are present in the cochlea, the semicircular canals, and the otolith organs. ${ }^{+12}$ Thus, an immune response directed against melanocytes may well elicit labyrinthine inflammation involving both the cochlear and vestibular nerves.

Defective smooth pursuit movements with a reduced gain strongly suggest central nervous system involvement, such as lesion of the brainstem, ${ }^{1314}$ cerebellum, ${ }^{15-17}$ and cortex. ${ }^{18-20} \mathrm{~A}$ brainstem lesion is less likely, because it should produce lower VOR gain, impaired optokinetic nystagmus and impaired saccades. ${ }^{21}$ The cerebellum is involved in visual modulation of the $\mathrm{VOR}^{22}{ }^{23}$ and patients with cerebellar disease $^{151624}$ as well as monkeys with floccular lesions $^{2526}$ have impaired visual suppression of vestibular nystagmus. Impaired visual suppression is also very useful for making a diagnosis of multiple sclerosis. ${ }^{16}$ Patients with cerebral hemidecortication show abnormal visual vestibular interactions. ${ }^{18} 19$ However, the visual vestibular interactions were normal in our patients with VKH syndrome, and the saccadic latency time and trajectory were not affected. These results indicate that the cerebellum and cerebrum were functionally normal despite the occurrence of leptomeningitis. An acute labyrinthine lesion could cause a transient reduction of smooth pursuit gain in the direction of the spontaneous nystagmus. ${ }^{14}$ Accordingly, dysfunction of the integrator circuit for slow eye movements was probably due to impaired afferent input from the labyrinth rather than to a lesion of cerebellum or cerebrum.

The abnormality in $\mathrm{VKH}$ syndrome is peculiar because patients have elevated VOR gain despite the presence of a peripheral vestibular lesion, and the mechanism involved in this gain elevation continues to be difficult to define. It was not simply due to the superposition of spontaneous nystagmus onto VOR gain, because ipsilateral VOR gain was still elevated when the slow phase velocity of spontaneous nystagmus was subtracted from VOR $(p<0.05,0.32-$ $0.47 \mathrm{~Hz}$ ), and contralateral VOR gain was also elevated $(p<0.05)$. We can suggest two potential causes. The first is a central mechanism related to visual impairment. ${ }^{27}$ However, there was no significant difference in visual acuity between groups A and B. The second is excitation of the labyrinth, as is seen in the acute phase of Ménière's disease. ${ }^{28}$ Electrocochleography has shown similarities between Ménière's disease and VKH syndrome. ${ }^{29}$ Inflammation of the labyrinth may elevate the endolymphatic pressure or may alter the flow of endolymph soon after the onset of VKH syndrome. An increase in flow towards the ampulla without impairment of the function of the sensory hair cells may contribute to the elevation of VOR gain. The severity of the change may differ between the two sides 
and this asymmetry could lead to spontaneous nystagmus. Paresis of canal function may appear in an advanced stage of the disease and could account for the transient VOR deterioration in case 3 . We think that the more likely explanation is the second.

The differential diagnosis of uveitis is often clinically difficult. Serous retinal detachment with focal subretinal leakage from the choroid is the most important finding in the diagnosis of VKH syndrome. However, patients with posterior scleritis and disseminated multifocal pigment epitheliopathy often exhibit a very similar appearance. Cerebrospinal fluid pleocytosis is also not specific to VKH syndrome since it may also be present in patients with sarcoidosis, Behçet's disease, uveal effusion, and other conditions. However, vestibulo-ocular abnormalities - that is, elevated VOR gain, appear to be common in VKH syndrome, and $53 \%$ of our patients exhibited spontaneous nystagmus. These findings may thus be very useful in confirming a diagnosis of $\mathrm{VKH}$ syndrome.

1 Vogt A. Frühzeitiges Ergrauen der Zilien und Bemerkungen über den sogennanten plötzlichen Eintritt dieser
Veränderung. Klin Monatsbl Augenheilkd 1906; 44: 228-42.

2 Koyanagi Y. Dysakousia, Alopecia und Poliosis bei schwerer Uveitis nicht traumatischen Ursprungs. Klin Monatsbl Uveitis nicht traumatischen
Augenheilkd 1929; 82: 194-211.

3 Harada E. Clinical study of non-suppurative choroiditis. A report of acute diffuse choroiditis. Acta Soc Ophthalmol fpn 1926; 30: 351-77.

4 Sugiura S. Vogt-Koyanagi-Harada disease. Fpn $\mathcal{f}$ Ophthalmol 1978; 22: 9-35.

5 Cowper AR. Harada's disease and Vogt-Koyanagi syndrome: uveoencephalitis. Arch Ophthalmol 1951; 45: 367-76.

6 Levy NS, Kramer SG, Barros TD. Pupillary and accommodative abnormalities in the Vogt-Koyanagi-Harada syndrome. Am $\mathcal{F}$ Ophthalmol 1970; 69: 582-8.

7 Yamada T, Ukai K, Ishikawa S. Least square estimation of time constant using a microcomputer. Acta Soc Ophthalmol fpn 1988; 92: 344-50.
8 Takemori S. Visual suppression test. Ann Otol Rhinol Laryngol 1977; 86: 80-5.

9 Suzuki J, Komatsuzaki A. Clinical application of optokinetic nystagmus, optokinetic pattern test. Acta Oto-laryng 1964;

10 Leigh RJ, Zee DS. The neurology of eye movement. 2nd ed. Philadelphia: FA Davis, 1991: 378-534.

11 Chan CC, Palestine AG, Kuwabara T, Nussenblatt RB. Immunopathologic study of Vogt-Koyanagi-Harada syndrome. Am $\mathcal{F}$ Ophthalmol 1988; 105:607-11.

12 Borghesan E. Nature of a pigmented substance in the labyrinth. Acta Oto-laryng 1964; 57: 288-93.

13 Waespe W, Martin P. Pursuit eye movements in a patient with a lesion involving the vestibular nuclear complex. NeuroOphthalmology 1987; 7: 195-202.

14 Baloh RW, Honrubia V, Sills A. Eye tracking and optokinetic nystagmus. Ann Otol Rhinol Laryngol 1977; 86: 108-14.

15 Zee DS, Yee RD, Cogan DG, Robinson DA, Engel K. Ocular motor abnormalities in hereditary cerebellar ataxia. Brain 1976; 99: 207-34.

16 Sharpe JA, Goldberg HJ, Lo AW, Herishanu YO. Visualvestibular interaction in multiple sclerosis. Neurology 1981; 31: 427-33.

17 Troost BT, Del'osso LF, Daroff RB. Effect of visual pursuit deficit on the human vestibulo-ocular reflex [Abstract]. Neurology 1976; 26: 352-3.

18 Sharpe JA, Lo AW, Rabinovitch HE. Control of saccadic and smooth pursuit system after cerebral hemidecortication. Brain 1979; 102: 387-403.

19 Sharpe JA, Lo AW. Voluntary and visual control of the vestibuloocular reflex after cerebral hemidecortication. Ann vestibuloocular reflex after 1981 ; 10: $164-72$

20 Baloh RW, Yee RD, Honrubia V. Optokinetic nystagmus and parietal lobe lesion. Ann Neurol 1980; 7: 269-76.

21 Baloh RW, Honrubia V. Clinical neurophysiology of the vestibular system. Philadelphia: FA Davis, 1979.

22 Robinson DA. Adaptive gain control of vestibuloocular reflex by the cerebellum. $\mathcal{F}$ Neurophysiol 1976; 39: 954-69.

23 Ito $M$. Neural design of the cerebellar motor control system. Brain Res 1972; 40: 81-4.

24 Baloh RW, Jenkins HA, Honrubia V, Yee RD. Visualvestibular interaction and cerebellar atrophy. Neurology 1979; 29: 116-9.

25 Takemori S, Cohen B. Loss of visual suppression of vestibular nystagmus after flocculus lesions. Brain Res 1974; 72: 213 24

26 Zee DS, Yamazaki A, Butler PH, Gucer G. Effects of ablation of flocculus and paraflocculus on eye movements in primate. F Neurophysiol 1981; 46: 878-99.

27 Zee DS. New concept of vestibular nystagmus. In: Barber HO, Sharpe JA, eds. Vestibular disorders. Chicago: Year Book Medical Publishers, 1988: 189-200.

28 McClure JA, Copp JC, Lyctt P. Recovery nystagmus in Meniere's disease. Laryngoscope 1981; 91: 1727-37.

29 Nishimoto Y. Pathophysiological studies of the cochlea of Vogt-Koyanagi-Harada's disease. Otologia Fukuoka 1988; 34: 154-69. 